\title{
THE EFFICACY OF SIMVASTATIN DRUG INJECTION FOR REGENERATION OF INDUCED SMALL PERFORATED TMJ DISC. (AN EXPERIMENTAL STUDY)
}

\author{
Ashraf Ghanem*
}

\begin{abstract}
Objectives: The current study aimed to evaluate arthroscopically, macroscopically and histopathologically the efficacy of Simvastatin drug intra-discal injection for stopping degeneration and increasing regeneration of induced perforated TMJ disc.

Methodology: Ten goats (20 joints) were incorporated in the study. Stab perforations were performed in all TMJs' discs in both sides of each goat under arthroscopic guidance, on joints of all animals the left TMJ acted as a control group without injecting any solutions after disk perforation, whereas the right TMJ acted as the experimental group by injecting the simvastatin hydrogel in the perforation site.
\end{abstract}

Results: Arthroscopic, macroscopic and histopathological evaluation revealed that the injected perforation sites had higher scores (regeneration) than the non-injected perforation sites. The present study proved that injection of simvastatin hydrogel $5 \mathrm{mg} / \mathrm{ml}$ promotes autogenously chondrogenic disc repair and retard disc degeneration, which provides an alternative strategy for biological TMJ disc perforations repair in a less expensive and easily applied method.

Conclusion: the current study recommended that, arthroscopic simvastatin hydrogel injection can be safely conducted on perforated TMJ disc patients.

KEY WORDS: Simvastatin hydrogel injection, perforated TMJ disc, arthroscopic picture and disc regeneration.

\section{INTRODUCTION}

Temporomandibular joint (TMJ) anatomically is a di-arthrodial joint; an articulation of the mandible and glenoid fossa with intervening disc ${ }^{1,2}$. TMJ disc perforation (DP) is one of the most challenging TMJ disorder that most commonly caused by abnormal relation of the intra-articular disc to the condyle and temporal bone ${ }^{3}$. This entity state has been associated with internal derangement (ID) of the TMJ usually in cases of anterior disc displacement without reduction ${ }^{4,5}$. Moreover, previous reports had related DP to degenerative joint disease

\footnotetext{
* Associate Professor; Department of Oral \& Maxillofacial Surgery, Faculty of Dentistry, Minia University, Egypt.
} 
(DJD) or Osteoarthritis (OA) both Clinically ${ }^{5}$ and experimentally ${ }^{6,7}$. DP is seen in about $5-15 \%$ of (TMJ) with displaced discs on arthroscopic examination of patients in the late stage ${ }^{8-10}$.

Regarding the relation of the perforation site to the type of disc displacement Liu et $\mathrm{al}^{3}$ showed that disc perforation in medial part of disc frequently occurs in patients with anterolateral displacement. While the ratio of disc perforation in medial or lateral part in patients with pure anterior displacement and antro-medial displacement was $96.0 \%$ and $100 \%$ respectively.

Zhang et al. in 2009 had published that intradiscal cholesterol lowering drug (Simvastatin drug) injection had been evolved as a new modality for regeneration of intervertebral disc (IVD) in a rat model of degenerative disc disease (DDD) ${ }^{11}$.

Another study in $2014^{12}$ had concluded that degenerated IVDs that had been injected with $5 \mathrm{mg} / \mathrm{mL}$ simvastatin drug in a hydrogel carrier demonstrated histologic features resembling normal and non-injured IVDs.

Accordingly, it will be of interest to evaluate experimentally simvastatin intra-discal injection in the TMJ field on an animal model by performing a stab injury in TMJ discs using a full thickness needle puncture injury under endoscopic guidance for making an animal model having TMJ DP.

\section{MATERIAL AND METHODS}

Ten healthy mature goats had been chosen of the same race, age, weight (12:26 kg). All animals' TMJs were arthroscopically examined to assure that all joints were normal. They were housed in an isolated building located as far away from dust, smoking, wild rodents and insects as possible. A balanced diet was introduced containing protein, carbohydrates, fat, minerals, vitamins and water. A customized wooden box provided with proper height, width and depth provided with wheels, it was fabricated for easy animal transportation protection from transit infections, injuries and stress.

Poly ethylene glycol hydrogel(PEG) OXFORD ${ }^{\circledR}$ ) was prepared by thermo stirring PEG powder (molecular weight $=6000)$ with saline using (Hot plate Stirrer) device. Simvastatin (Zocor ${ }^{\circledR}$ MSD) was added to the (PEG) gel with a specific weight to reach the concentration of $5 \mathrm{mg} / \mathrm{ml})^{*}$.

\section{The surgical procedures}

Under general anesthesia, the pre-auricular region on both sides was shaved, scrubbed, and sterilized with Povidone-iodine (Betadine $^{\circledR}$ ) antiseptic solution. Surface Anatomical Landmarks were marked on the goat heat as showed in figure 1 .

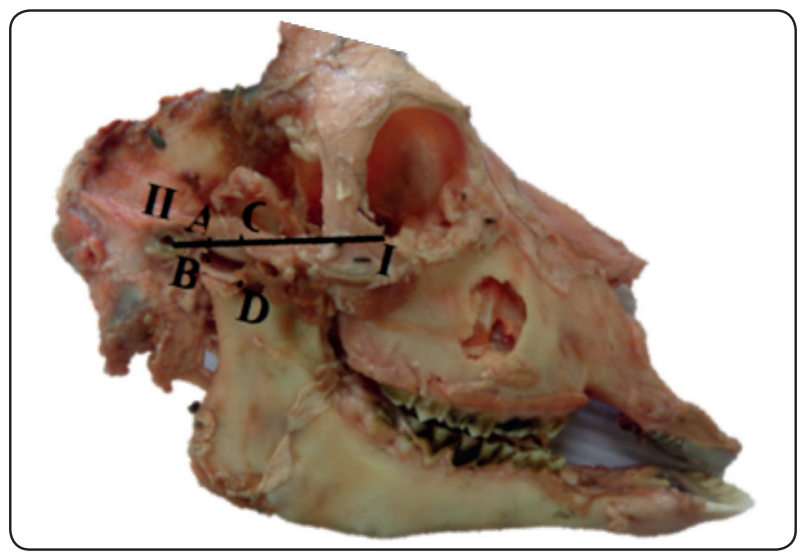

Fig. (1) Dissected goat head for illustration of the surface anatomic landmarks for making an easy arthroscopic TMJ examination.

The study was surgically incorporated into two stages. The first stage was to perform disc perforation under General Anesthesia (G.A.) using the arthroscopic guidance via $18 \mathrm{G}$ needle on both TMJs of each goat, in order to simulate TMJ disc perforation on an animal model. In all animals the left TMJ acted as a control group without injecting any solutions following the disc perforation, whereas the right TMJ acted as the experimental group by injecting the simvastatin hydrogel in the perforation site as showed in figure 2 . 


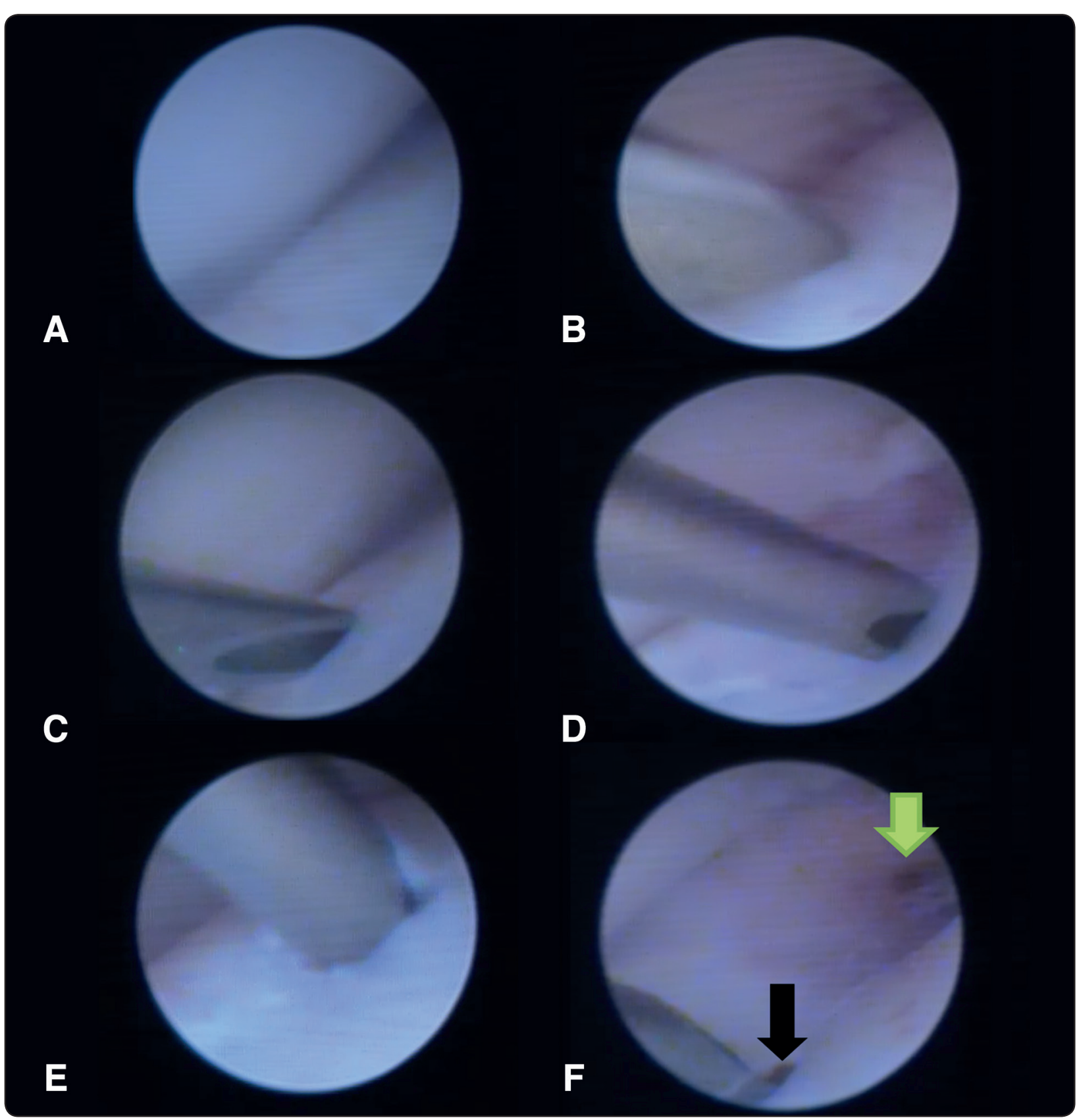

Fig. (2) The arthroscopic surgical procedures A: normal arthroscopic TMJ white to white appearance. B: insertion of a 18 Gauge needle through the $2^{\text {nd }}$ puncture. $\mathbf{C}$ and $\mathbf{D}$ : performing stab perforation through the superior joint space via the $2^{\text {nd }}$ puncture. E: rotation of the needle (180) degree and held in place from (5-30) seconds. F: green arrow and black arrows showing repeated punctures. 
The second stage was performed under G.A using the same standardized arthroscopic technique to evaluate the healing of the perforation sites on both joints. This stage was started after 4-6 weeks from the 1 st stage to allow enough time for disc regeneration.

Following arthroscopic evaluation euthanization was done by increasing the dose of G.A to a lethal level. Immediate open surgical arthrotomy was performed to excise TMJ Discs with 3-4 mm from the surrounding articular structures. Then macroscopic evaluation of healing in the perforation sites Thereafter discs' specimen were prepared and submitted for histological evaluation.

\section{Statistical analysis}

The results of endoscopic, macroscopic and histologic evaluations were properly tabulated using (Statistical Package for the Social Sciences (SPSS) program edition 22 for performing the statistical analysis). The individual student $\mathrm{T}$ test was used for comparison of the degeneration and the regeneration arthroscopic, macroscopic and histological findings within each group after 4:6 weeks of the disc perforation and injection for showing the statistical significance of simvastatin drug effect on the regeneration of TMJ disc perforation.

\section{RESULTS}

All animals successfully sustained the surgical procedures without major or minor complications. All goats were able to load their TMJs postoperatively without any dysfunctions. They continued their standard nutrition during the healing period. No evidence of surgery related complications (e.g., joint or surgery site infection) was observed. No signs of adverse effects related to Simvastatin drug injection were detected.

\section{Arthroscopic evaluation}

Evaluation of the arthroscopic features of all perforations using a modified OAS at the $2^{\text {nd }}$ surgical stage (i.e. after 4:6 weeks of injection) revealed that arthroscopically injected perforations with $5 \mathrm{mg} / \mathrm{mL}$ of Simvastatin drug in a hydrogel carrier $1.5 \mathrm{ml}$ had higher O'Swestry arthroscopic score (OAS) than the non-injected perforation sites (mean a greater regeneration happened in SIM-injected perforations) as showed in the attached table.

The SIM-injected perforation sites had a cartilaginous formation that were in the level with the surrounding normal cartilage in $80 \%$ of specimens while the last $20 \%$ were showing healing that was below the level of the surrounding cartilage. On the other hand, the non SIM-injected perforations

TABLE (1) Comparison between the mean OAS of all SIM-injected perforation sites and the mean OAS of all Non SIM-injected perforation sites, $\mathrm{p}$-value and $\mathrm{t}$-value of paired student-t-test for comparison between the two means.

\begin{tabular}{|l|c|c|c|c|}
\hline TYPE Of SPECIMEN & MEAN & SD VALUE & T-VALUE & P-VALUE \\
\hline $\begin{array}{l}\text { The mean OAS of SIM-injected } \\
\text { perforation sites }\end{array}$ & 9.2000 & 1.09545 & 16.738 & 0.000 \\
\hline $\begin{array}{l}\text { The mean OAS of non, injected } \\
\text { Perforation sites }\end{array}$ & 1.0000 & 0.00000 & & \\
\hline
\end{tabular}

Significant at $P \leq 0.05$ 
hadn't cartilaginous formation at all or have healing that was below the level of the surrounding cartilage in $10 \%$ of specimens. The injected perforations healing tissues were completely integrated with the surrounding cartilage in $80 \%$ of specimens while the last $20 \%$ had minor disruption ( $<25 \%$ of area).

Concerning the color of all injected perforation sites were Pearly, hyaline-like. On the other hand the color of the non-injected perforation sites had a yellowish color in $80 \%$ of the perforations and the last $20 \%$ of the specimens had a whitish color.

\section{Macroscopic evaluation}

Evaluating the macroscopic degree of defect repair in SIM-injected perforations the healing were in level with surrounding normal cartilage in $80 \%$ of perforation sites and the repair was in level of $75 \%$ of defect depth in the remaining $25 \%$ perforation sites. On the other hand non-SIM injected perforations had no repair on all the defect depth in $60 \%$ of perforation sites and the remaining $40 \%$ had $25 \%$ repair of defect depth. Macroscopic assessment was more accurate than arthroscopic assessment because it enabled us to inspect the excised TMJ discs from its superior and inferior articulating surfaces.
Concerning the integration of cartilaginous repair to border zone the healing occurred in SIMinjected perforations had complete integration with surrounding cartilage in $80 \%$ of perforation sites and there was demarcating border $<1 / 4$ of the perforation size in $20 \%$ of disc perforations. On the other hand non SIM-injected disc perforations had no contact between the borders of the perforation in $40 \%$ of specimens however the last $60 \%$ of perforations were integrated in half of their borders. These results were comparable to the arthroscopic assessment results of borders integrations however the macroscopic assessment revealed 20\% increase in borders integrations of the non-injected perforation sites when they were inspected from the inferior articulating surface of TMJ disc.

Regarding evaluating the macroscopic appearance of perforation sites of SIM-injected TMJs had intact smooth surface in $20 \%$ of perforation sites, fibrillated surface appearances in $40 \%$ of perforations and there were $40 \%$ having Small, scattered fissures or cracks. On the other hand $60 \%$ of non-SIM injected perforations had appearance of several large fissures. The last $40 \%$ where had macroscopic appearance of total degeneration. These results were comparable to the arthroscopic assessment of the perforation sites surface appearance as showed in Figure 3.

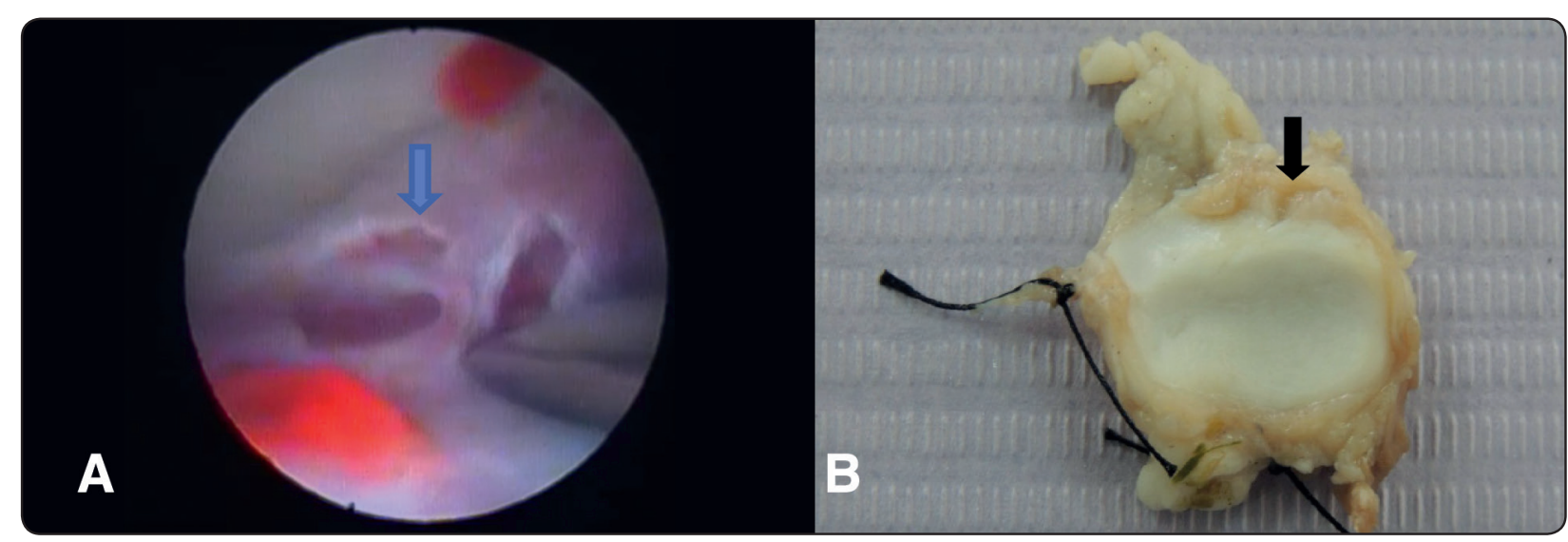

Fig. (3) A: At stage (I) blue arrow showing arthroscopic picture of through and through disc perforation trans the posterior discal attachment and the posterior part of the disc. It was injected with saline, B: Gross picture at stage (II) after 4:6 weeks black arrow showing no healing had been occurred neither in the posterior attachment nor in the posterior part of the disc. 


\section{Histologic evaluation}

All SIM-injected perforations were showing Freedom from cellular changes of degeneration or hypo cellularity and were showing normal cellularity, whereas $70 \%$ of non-SIM injected perforations were showing severe hypo cellularity and the last $30 \%$ were showing moderate hypo cellularity

All SIM-injected perforations were showing freedom from degenerative changes in adjacent cartilage. Their adjacent cartilages were showing normal cellularity, no clusters and were having normal staining. On the other hand in adjacent cartilages of non-SIM injected perforations $60 \%$ were showing mild or moderate hypo cellularity and slight staining. The last $40 \%$ of specimens were showing normal cellularity, mild clusters and moderate staining as showed in Figure 4.

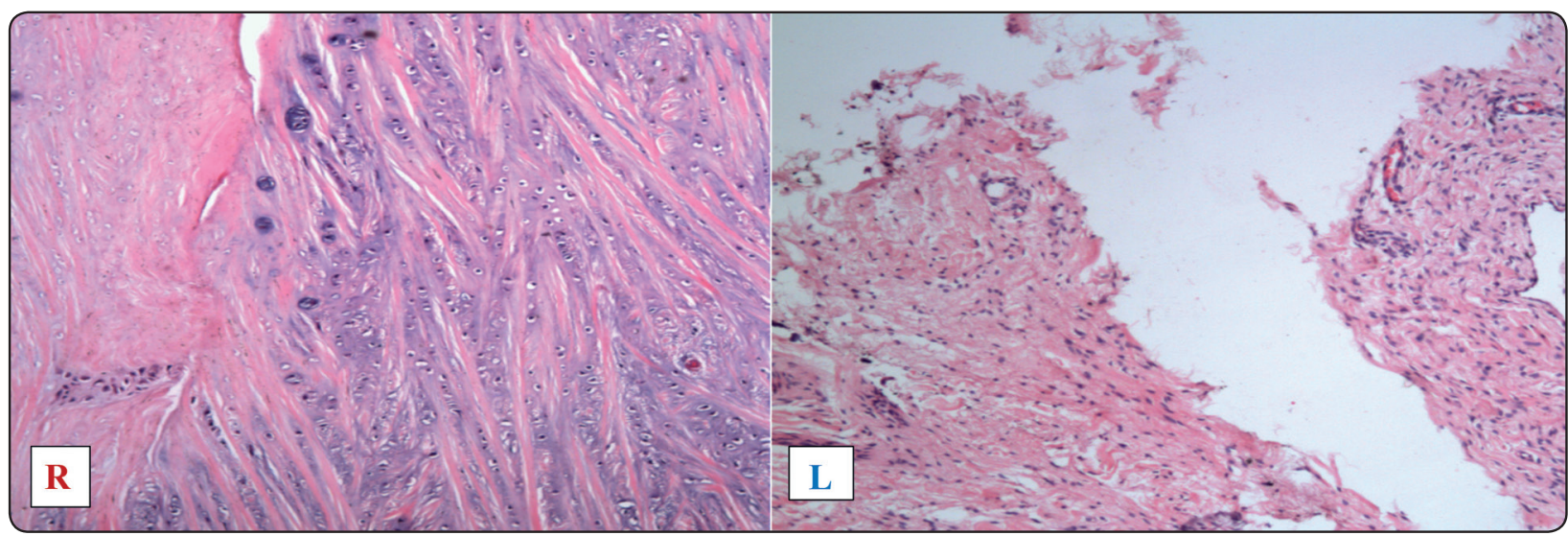

Fig. (4) The histological appearance of sagittal longitudinal section of the perforated TMJs' discs of the 1st goat. (R): Shows cells adjacent to the perforation are chondrocytes like cells within its lacunae with formation of reparative hyaline and fibro cartilage. (L): cells adjacent to the non-injected perforation are fibrous like cells without formation of any reparative hyaline or fibro cartilage (original magnification x100).

\section{DISCUSSION}

TMJ disc possesses no mesenchymal cell mediated regenerative or adaptive capacity ${ }^{11}$. Therefore a gradual abrasive loss of discal tissues cannot be compensated and will eventually lead to perforation of the thinnest disc region. Consequently, searching for a local drug that can hinder TMJ disc degeneration and increase disc regeneration remains an important goal for many researchers.

Although previous studies ${ }^{11,12}$ were proposing that simvastatin drug in hydrogel $5 \mathrm{mg} / \mathrm{ml}$ injection can improve the regeneration capacity of the degenerated IVDs. Many reasons had encouraged us to test Simvastatin drug application effectiveness experimentally on regeneration of perforated TMJ disc for its good results in regeneration property of degenerated discs (IVDs), its availability and its minimal-invasive injection procedures. Many authors had found that we can simulate an animal model of DDD by needle puncture to the articular $\operatorname{disc}^{13 \sim 15}$.

Up to our knowledge the current study was the first study experimentally examined the efficacy of Simvastatin hydrogel on regeneration of the stabbed perforated TMJ disc. The research on line or even in the literatures does not mention that regard and a the application to the TMJ disk 
Regarding the recommended dose of simvastatin drug injection; Than et al. ${ }^{12}$ in 2014 demonstrates greater benefit of $5 \mathrm{mg} / \mathrm{ml}$ simvastatin in hydrogel than higher doses of $10 \mathrm{mg} / \mathrm{ml}$ and $15 \mathrm{mg} / \mathrm{ml}$. They claimed that higher doses were toxic to the disc secondary to altered cell membrane adipose metabolism, resulting in cell death. Other studies have found that higher doses of statins decrease cell viability in a variety of cell types ${ }^{17}$ due to interference of farnesyl pyrophosphate and geranyl pyrophosphate, which are involved in control of cell adhesion, growth, and survival through protein prenylation ${ }^{18,19}$. Therefore the larger doses (10 $\mathrm{mg} / \mathrm{ml}$ and $15 \mathrm{mg} / \mathrm{ml}$ ) that were injected in saline (were absorbed rapidly) were had bad results on the cartilaginous regeneration.

Concerning the assessment methods of the current study we had used three different standardized evaluation techniques using arthroscopic, macroscopic and histologic assessment methods this revealed broader assessment methods.

The arthroscopic results were comparable to the results of Simvastatin previous studies 11, 12 which assessed the cartilaginous regeneration by measuring the overall thickness of the stabbed injected and non-injected IVDs on MRI. Our study had more precise assessment than the previous studies due to evaluating the cartilaginous healing volumes using the direct arthroscopic visualization on the same sites of perforations. However previous studies were had less precise results as they didn't measure the cartilaginous healing of perforations sites but they measured overall thickness of the IVDs on MRI.

Concerning the macroscopic degree of defect repair in SIM-injected perforations the healing were in level with surrounding normal cartilage in $80 \%$ of perforation sites and the repair was in level of $75 \%$ of defect depth in the remaining $20 \%$ perforation sites.

The overall assessment revealed that all SIMinjected perforations where having superior than the non-injected perforations. This means a good capability of simvastatin to cause higher regeneration of TMJ disc fibrocartilages which was documented by our results.

\section{CONCLUSION}

The present study proved that injection of simvastatin drug hydrogel $5 \mathrm{mg} / \mathrm{ml}$ promotes autogenously chondrogenic disc repair and retard disc degeneration, which provides an alternative strategy for biological TMJ disc perforations repair in a less expensive and easily applied method and it is recommended that arthroscopic simvastatin drug hydrogel injection can be safely conducted on perforated TMJ disc patients.

\section{ACKNOWLEDGEMENT}

The acknowledgement for the role of Prof. Khaled Barkat for providing his own endoscope in performing some of the practical endoscopy, as well Mr. Ahmed Gamal for his collaboration.

\section{REFERENCES}

1- Gerard MW, Laughon MM, Colley JL, 3rd, Glasheen WP, Hoard MA, Edlich RF. Trends in temporomandibular joint surgery. Med Prog Technol 1996;21(4):171-5.

2- C. Fletcher M, Piecuch JF, Lieblich SE, editor. Anatomy and Pathophysiology of the temporomandibular Joint. 2 ed. Hamilton,Ontario: BC Decker: 2004.

3- Liu XM, Zhang SY, Yang C, Chen MJ, X YC, Haddad $\mathrm{MS}$, et al. Correlation between disc displacements and locations of disc perforation in the temporomandibular joint. Dentomaxillofac. Radiol 2010 Mar;39(3):149-56.

4- Kondoh T, Westesson PL, Takahashi T, Seto K. Prevalence of morphological changes in the surfaces of the temporomandibular joint disc associated with internal derangement. J Oral Maxillofac Surg 1998 Mar;56(3):33943; discussion 43-4.

5- Cholitgul W, Petersson A, Rohlin M, Akerman S. Clinical and radiological findings in temporomandibular joints with disc perforation. Int J Oral Maxillofac Surg 1990 Aug;19(4):220-5. 
6- Helmy E, Bays R, Sharawy M. Osteoarthrosis of the temporomandibular joint following experimental disc perforation in Macaca fascicularis. J Oral Maxillofac Surg 1988 Nov; 46(11):979-90.

7- Scapino. Histopathology associated with malposition of the 22 human temporomandibular joint discs. Oral Surg Oral Med Oral Pathol 1983;2(2):55:38.

8- Yura S, Nobata K, Shima T. Diagnostic accuracy of fatsaturated T2-weighted magnetic resonance imaging in the diagnosis of perforation of the articular disc of the temporomandibular joint. Br J Oral Maxillofac Surg 2011 Jun; 50(4):365-8.

9- Dimitroulis G. The prevalence of osteoarthrosis in cases of advanced internal derangement of the temporomandibular joint: a clinical, surgical and histological study. Int J Oral Maxillofac Surg 2005 Jun; 34(4):345-9.

10- Lee JY, Kim DJ, Lee SG, Chung JW. A longitudinal study on the osteoarthritic change of the temporomandibular joint based on 1-year follow-up computed tomography. J Craniomaxillofac Surg 2011 Dec; 40(8):e223-8.

11- Zhang H, Wang L, Park JB, Park P, Yang VC, Hollister $\mathrm{SJ}$, et al. Intradiscal injection of simvastatin retards progression of intervertebral disc degeneration induced by stab injury. Arthritis Res Ther 2009;11(6):R172.

12- Than KD, Rahman SU, Wang L, Khan A, Kyere KA, Than TT, et al. Intra-discal injection of Simvastatin results in radiologic, histologic, and genetic evidence of disc regeneration in a rat model of degenerative disc disease. Spine J 2014 Jun 1; 14(6):1017-28

13- Masuda K, Aota Y, Muehleman C, Imai Y, Okuma M, Thonar EJ, et al. A novel rabbit Model of mild, reproducible disc degeneration by an annulus needle puncture: correlation between the degree of disc injury and radiological and histological appearances of disc degeneration. Spine (Phila Pa 1976) 2005 Jan 1; 30(1):5-14.

14- Issy AC, Castania V, Castania M, Salmon CE, NogueiraBarbosa $\mathrm{MH}, \mathrm{Bel} \mathrm{ED}$, et al. Experimental model of intervertebral disc degeneration by needle puncture in Wistar rats. Braz J Med Biol Res 2013 Mar; 46(3):235-44.

15- Kwon YJ. A minimally invasive rabbit model of progressive and reproducible disc degeneration confirmed by radiology, gene expression, and histology. J Korean Neurosurg Soc 2013 Jun; 53(6):323-30.

16- Munoz-Guerra MF, Rodriguez-Campo FJ, Escorial Hernandez V, Sanchez-Acedo C, Gil-Diez Usandizaga JL. Temporomandibular joint disc perforation: long-term results after operative Arthroscopy. J Oral Maxillofac Surg 2012 Apr; 71(4):667-76.

18- Dirks AJ, Jones KM. Statin-induced apoptosis and skeletal myopathy. Am J Physiol Cell Physiol 2006 Dec; 291(6):C1208-12.

19- Aznar S, Lacal JC. Rho signals to cell growth and apoptosis. Cancer Lett 2001 Apr 10; 165(1):1-10. 